\title{
Indicadores de calidad en la valoración de intestino delgado mediante cápsula endoscópica en un centro de tercer nivel
}

\author{
Luis F. García-Contreras*, Gerardo Blanco-Velasco, Omar M. Solórzano-Pineda y \\ Óscar V. Hernández-Mondragón
}

Departamento de Endoscopia Gastrointestinal, Hospital de Especialidades, Centro Médico Nacional Siglo XXI, Instituto Mexicano del Seguro Social, Ciudad de México, México

\begin{abstract}
Resumen
Introducción: Los indicadores de calidad (IC) estandarizan los procedimientos endoscópicos para brindar mejores resultados. La Sociedad Europea de Endoscopia Gastrointestinal recientemente publicó IC para la valoración del intestino delgado (ID) mediante cápsula endoscópica (CE). Objetivo: Valorar el apego a los IC sugeridos en la evaluación del ID mediante $C E$ en un centro de tercer nivel. Métodos: Se realizó una revisión e inclusión de las CE realizadas en nuestro centro durante 18 meses. Resultados: Se incluyeron 200 CE, dividiendo los IC en preprocedimiento, intraprocedimiento y posprocedimiento, así como mayores y menores. De los preprocedimiento se excluyó la valoración de pacientes con alto riesgo de retención de CE mediante Patency por no contar con esta; se realizó la CE por una indicación adecuada y no se cumplió con realizar la CE de forma temprana en aquellos con hemorragia de ID. De los IC intraprocedimiento se logró una visualización completa del ID, se utilizó la terminología estructurada para CE y la lectura de la CE se realizó a 15 campos por segundo, fallando en la evaluación del grado de limpieza intestinal mediante escalas validadas al no documentarse en el reporte endoscópico. De los posprocedimiento la tasa de retención de CE fue adecuada y no se realizó un envío de forma adecuada a enteroscopia asistida por dispositivos. Cumpliendo por lo tanto con 4 de 6 IC clasificados como mayores y 2 de 3 clasificados como menores. Conclusiones: Se cumplieron las metas establecidas en 6 de 9 IC evaluados. La retroalimentación mediante la valoración de IC permite evaluar puntos débiles para mejorar estos en el futuro.
\end{abstract}

Palabras clave: Indicadores de calidad. Cápsula endoscópica. Intestino delgado.

\section{Performance measures in the evaluation of small bowel by capsule endoscopy on a third level hospital}

\section{Abstract}

Introduction: Quality indicators (QI) standardize endoscopic procedures to provide better results. The European Society for Gastrointestinal Endoscopy recently published QI for capsule endoscopy (CE) assessment of the small intestine (SI). Objective: To assess the adherence to the QI suggested in the evaluation of the SI by CE in a tertiary care center. Material and methods: $A$ review and inclusion of the CE performed in our center for 18 months was performed. Results: Two hundred CE were included, dividing the QI in pre-, intra- and postprocedures and into key and minor QI. The assessment of 
patients with a high risk of CE retention by Patency capsule was excluded from the pre-procedures because we did not have it; CE was performed by adequate indication and CE was not performed early in those with SI bleeding. From the intraprocedural QIs, complete visualization of the SI was achieved, structured terminology for endoscopic capsule was used and the CE reading was performed at 15 fields per second, failing to evaluate the degree of intestinal cleaning using validated scales because they were not documented in the endoscopic report. The CE retention rate was adequate in the postprocedures, but device-assisted enteroscopy was not adequately sent to all those in need. Therefore, there was compliance with 4 of the 6 QIs classified as major and 2 of 3 classified as minor. Conclusions: The goals established in 6 out of 9 QIs evaluated were met. Feedback via QI evaluation allows evaluating weak points for future improvement.

Key words: Quality indicators. Capsule endoscopy. Small intestine.

\section{Introducción y objetivo}

Millones de pacientes se someten a procedimientos endoscópicos al año, entre estos la cápsula endoscópica (CE); si bien es cierto que son un pilar en el diagnóstico y tratamiento de patologías gastrointestinales, no están exentos de riesgos, debiéndose realizar en el paciente, en el momento y con la técnica adecuada para obtener el mayor costo/beneficio. Bajo esta premisa las sociedades internacionales desarrollaron una forma de estandarizar los procedimientos mediante la creación de indicadores de calidad (IC), estos permiten brindar mayor seguridad, mejores resultados y experiencia a los pacientes; para esto los centros endoscópicos deben llevar un registro de estos marcadores para comparar el desempeño obtenido con las recomendaciones y cortes establecidos por sociedades endoscópicas y así realizar retroalimentación, mejorar la calidad de los procedimientos endoscópicos y asegurar la competencia de los endoscopistas. Los IC tienen que ser claros, objetivos, realistas, reproducibles, fáciles de medir y basados en evidencia, categorizándose como medidas estructurales, de proceso y de resultado, dividiéndose también en relación al periodo del estudio endoscópico en preprocedimiento, intraprocedimiento y posprocedimiento, así como en mediciones globales del centro e individuales ${ }^{1}$. Existen IC establecidos para la mayoría de procedimientos (colonoscopia, panendoscopia y colangiopancreatografía retrógrada endoscópica); sin embargo, no se habían establecido para la CE. No obstante, en 2017 la Sociedad Coreana de Endoscopia Gastrointestinal publicó 16 IC pre, intra y post realización de CE, sin embargo no dictaminó cortes específicos para estos y posteriormente la Sociedad Europea de Endoscopia Gastrointestinal (ESGE) publicó sus propios IC, los cuales incluyen también medidas estructurales y de resultado, con la ventaja de especificar los cortes mínimos a alcanzar en cada uno de los IC propuestos (Tabla 1) 2,3. Debido a esto se decidió realizar un estudio retrospectivo analizando las CE realizadas en nuestro centro de enero de 2018 a
Tabla 1. Indicadores de calidad para la valoración de CE y sus metas optimas y mínimas establecidas por la ESGE

\begin{tabular}{|l|c|c|}
\hline \multicolumn{2}{|c|}{ Indicadores de calidad en la valoración de cápsula intestinal } \\
\hline Indicador por valorar & $\begin{array}{c}\text { Meta } \\
\text { óptima }\end{array}$ & $\begin{array}{c}\text { Meta } \\
\text { mínima }\end{array}$ \\
\hline $\begin{array}{l}\text { Indicadores de calidad mayores } \\
\text { Indicación adecuada }\end{array}$ & $\geq 95 \%$ & $\geq 95 \%$ \\
\hline $\begin{array}{l}\text { Envío adecuado a EAD } \\
\text { Tasa de retención de CE }\end{array}$ & $\geq 90 \%$ & $\geq 75 \%$ \\
\hline Visualización completa del ID & $<2 \%$ & $<2 \%$ \\
Tasa diagnóstica de CE & $\geq 95 \%$ & $\geq 80 \%$ \\
\hline Tiempo de envío a CE en HTDM & $\geq 50 \%$ & $\geq 50 \%$ \\
\hline $\begin{array}{ll}\text { Indicadores de calidad menores } \\
\text { Uso de CEST }\end{array}$ & $\geq 90 \%$ & $\geq 90 \%$ \\
\hline $\begin{array}{l}\text { Velocidad de lectura adecuada } \\
\text { Detección de pacientes con riesgo de } \\
\text { retención de CE }\end{array}$ & $\geq 95 \%$ & $\geq 95 \%$ \\
Documentación de limpieza intestinal & $\geq 95 \%$ & $\geq 95 \%$ \\
\hline
\end{tabular}

CE: cápsula endoscópica; ID: intestino delgado; CEST: terminología estructurada para cápsula endoscópica; EAD: enteroscopia asistida por dispositivos; HTDM: hemorragia de tubo digestivo medio; ESGE: Sociedad Europea de Endoscopia Gastrointestinal.

junio de 2019 con el fin de evaluar el apego a los IC establecidos por la ESGE recientemente.

El objetivo de este estudio es valorar el apego a los IC sugeridos por la ESGE para la evaluación de intestino delgado (ID) mediante el uso de CE en nuestro centro de endoscopia de tercer nivel.

\section{Material y métodos}

Se realizó una revisión de las CE realizadas en el Departamento de Endoscopia del Hospital de Especialidades Dr. Bernardo Sepúlveda Gutiérrez del Centro Médico Nacional Siglo XXI, de enero de 2018 a junio de 2019, obteniendo un total de $200 \mathrm{CE}$, todas estas realizadas utilizando la cápsula PillCam SB3 (Given Imaging, Yokneam, Israel) con el software RAPID de lectura. Se revisó cada una de las 200 CE con el fin de valorar el apego a 9 de los 10 IC establecidos por la 


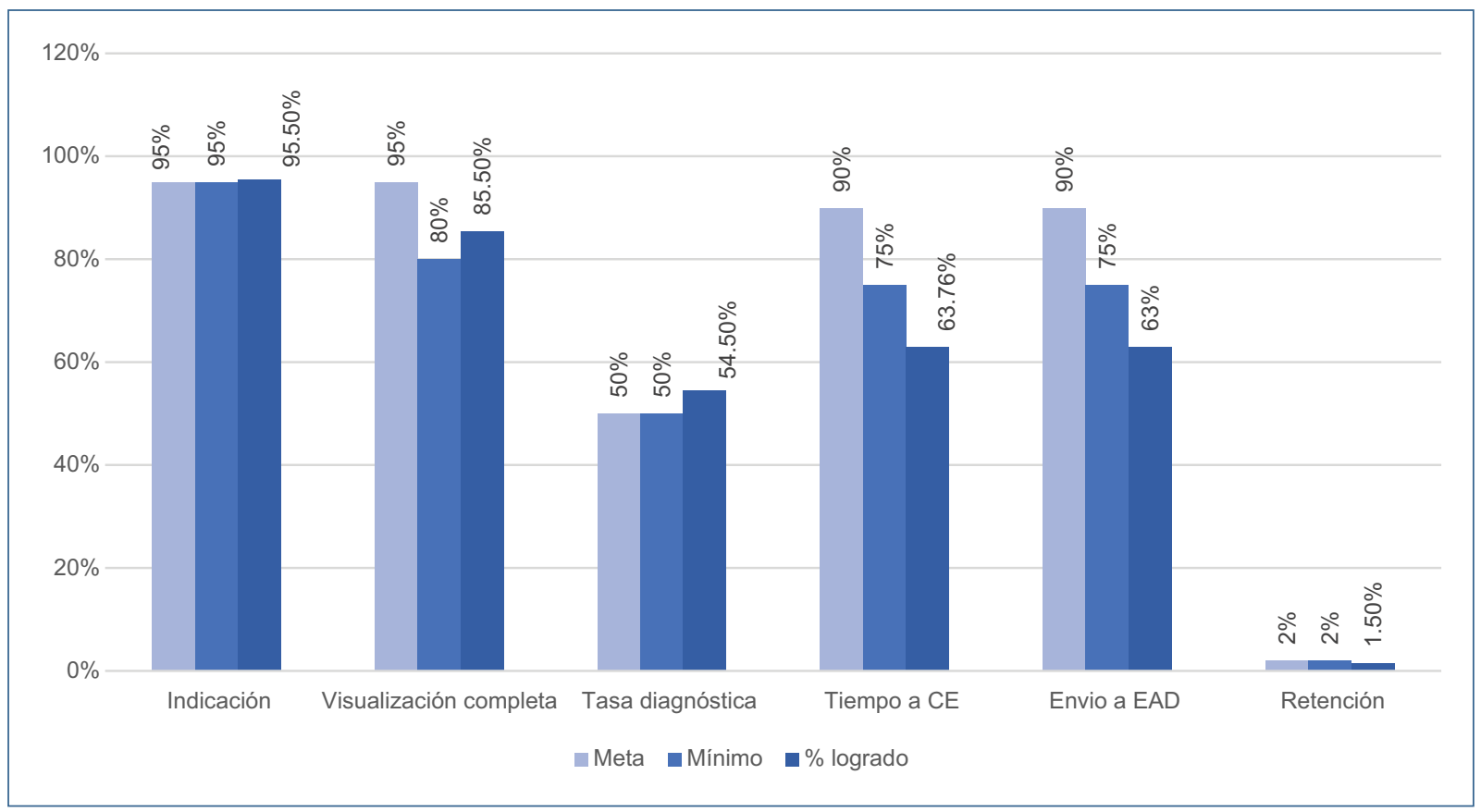

Figura 1. Cumplimiento de los indicadores de calidad mayores.

CE: cápsula endoscópica; EAD: enteroscopia asistida por dispositivos.

ESGE (Tabla 1), siendo el envío a cápsula Patency de pacientes con alto riesgo de retención el IC excluido de la valoración al no contar con dicho insumo en nuestro instituto. Se revisó minuciosamente cada reporte de $\mathrm{CE}$ y se dividieron los IC para facilitar su evaluación en preprocedimiento, intraprocedimiento y posprocedimiento, así como en mayores y menores; registrando el apego a cada uno de estos en una base de datos para su integración y evaluación posterior.

\section{Criterios de inclusión}

Se incluyeron todas las cápsulas endoscópicas para valoración de ID sin importar la indicación realizadas en el periodo de enero de 2018 a junio de 2019.

\section{Criterios de exclusión}

Se excluyeron aquellas cápsulas cuya lectura no fue posible por fallas técnicas de transmisión o aquellas en las que el paciente decidiera la retirada temprana del receptor de imagen.

\section{Resultados}

Se evaluaron 9 de los 10 IC establecidos por la ESGE, excluyendo la identificación de pacientes con alto riesgo mediante Patency al no tener disponibilidad de esta. Dividiendo los IC en preprocedimiento (indicación adecuada y tiempo de envío adecuado a CE en un episodio de hemorragia de tubo digestivo medio [HTDM]), en IC intraprocedimiento (evaluación de la limpieza intestinal, visualización completa de ID, tasa diagnóstica, uso de terminología estructurada para CE [CEST] y velocidad de lectura) y posprocedimiento (envío adecuado a enteroscopia asistida por dispositivos [EAD] y tasa de retención de $\mathrm{CE}$ ); así mismo dividiendo todos estos en IC mayores y menores, como se estableció por la ESGE. De todos estos IC evaluados hubo un cumplimiento con las metas establecidas en 6 de 9 IC, 4 de estos mayores y 2 menores (Figs. 1 y 2). Con cumplimiento de una indicación adecuada de la CE del $95.5 \%$, una visualización completa del ID en el $85.5 \%$ de las CE, así como una tasa diagnóstica del $54.5 \%$, utilización de CEST en la creación de los reportes en el $92 \%$ de los casos, una retención de la CE en solo el $1.5 \%$ de los casos y así mismo se realizó una lectura a velocidad adecuada en el $100 \%$ de los casos. Fallando en un envío temprano (< 14 días) a CE durante HTDM, al obtener cifras del $63.7 \%$, así como en la valoración de la limpieza intestinal al obtener un $0 \%$ del cumplimiento y por último se falló en lograr un envío adecuado a EAD al lograr cifras solo del $63.01 \%$. 


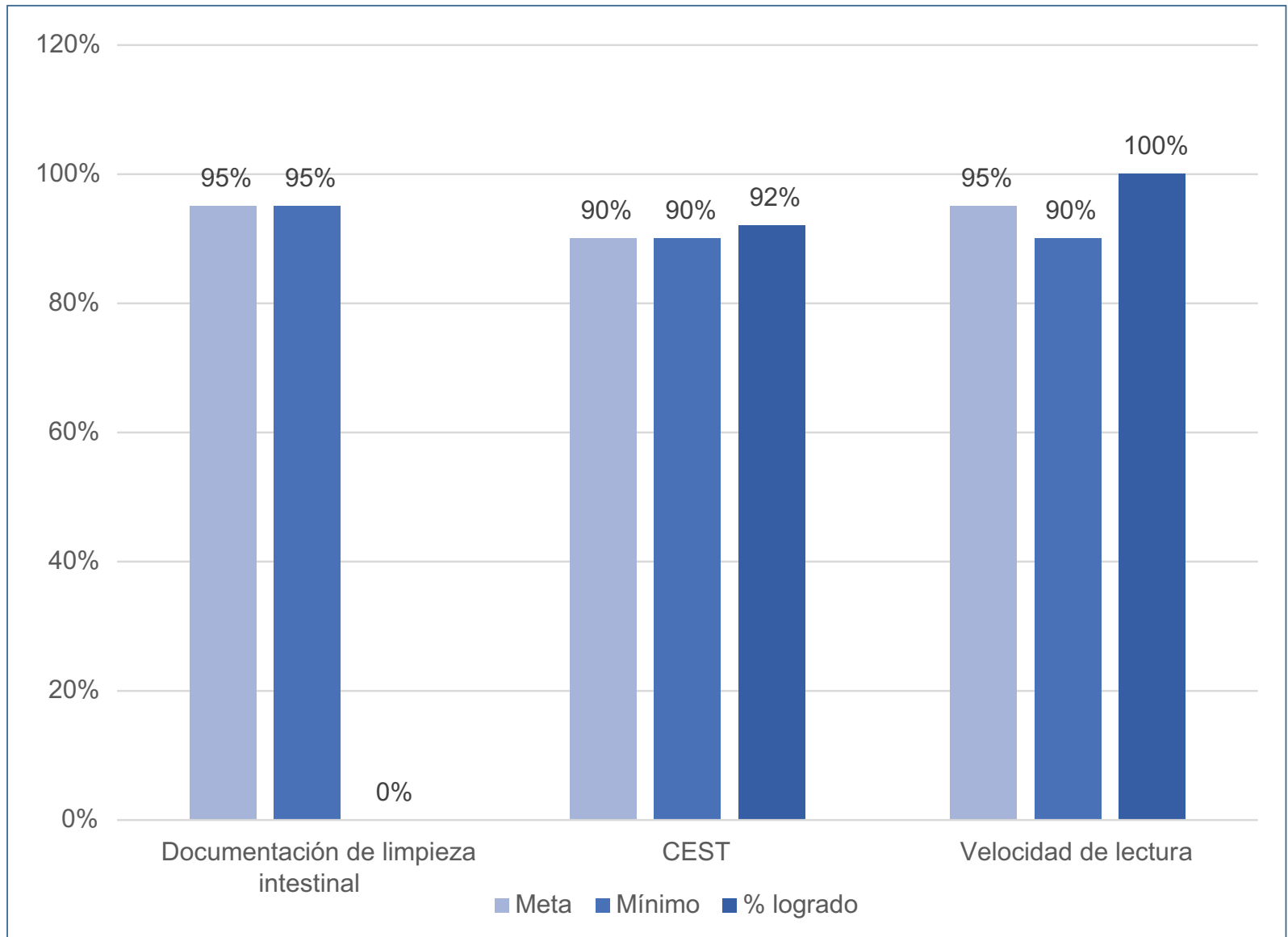

Figura 2. Cumplimiento de los indicadores de calidad menores. CEST: terminología estructurada para cápsula endoscópica.

\section{Discusión}

Los IC ayudan a evaluar y realizar los procedimientos endoscópicos de manera estandarizada, permitiendo realizar retroalimentación, mejorar la calidad y asegurar la competencia de los endoscopistas. En este estudio se valoraron los IC establecidos por la ESGE para la evaluación de CE logrando cumplir con las metas en 6 de los 9 IC evaluados. Dentro de los IC con los que se logró cumplir, 4 fueron clasificados como mayores y 2 como menores. Se cumplió con una indicación adecuada, siendo esto importante, ya que una indicación incorrecta se asocia a menores tasas diagnósticas y somete al paciente a un procedimiento injustificado con los potenciales riesgos intrínsecos a este; dentro de las indicaciones más comunes se encuentra la HTDM, anemia ferropénica, enfermedad de Crohn (EC) ya diagnosticada o sospecha de esta, síndromes de poliposis, tumores intestinales y enfermedad celiaca complicada 0 refractaria; otras indicaciones son controvertidas y el uso de CE en estas se tiene que justificar'; en nuestro centro las indicaciones más comunes fueron HTDM y búsqueda de tumores. Una valoración completa del ID se llevó de forma adecuada en la mayoría de los pacientes y se documentó tanto por escrito como por fotodocumentación, como es requerido ${ }^{3}$. En cuanto a la tasa diagnóstica de la CE se logró una tasa aceptable, del $54.5 \%$, recordando que la CE tiene rendimiento diagnóstico variable según la indicación por la que se realice; en HTDM las tasas diagnósticas van del 35 al $77 \%{ }^{5}$, para sospecha de EC o enfermedad definida del 6 al $39 \%$; siendo para otras indicaciones variables. El cumplimiento con la creación de reportes de CEST es importante debido a que facilita la creación de reportes uniformes, la educación y el entrenamiento de los lectores. La CEST especifica claramente la estructura y contenido de un reporte endoscópico, el tipo de cápsula usada, fecha, nombre del lector, indicación de la CE e 
historia clínica del paciente con los datos más relevantes; en cuanto al reporte de los hallazgos, reporta si se completó el procedimiento, la calidad de la visualización de la mucosa y los hallazgos usando terminología recomendada, así como las recomendaciones a seguir según estos ${ }^{7}$. En cuanto a la velocidad de lectura se realizó a 15 cuadros por segundo (CPS) en modo de visión sencillo en el $100 \%$ de los casos; esto es importante, puesto que se ha visto que si bien existen varios modos de lectura (visión sencilla, visión dual y visión cuádruple), ninguno se ha declarado como superior a los demás; si bien algunos estudios han demostrado que el modo doble y cuádruple logran mayor detección de lesiones que el modo sencillo $(83,85 \text { y } 56 \% \text { sucesivamente) })^{8}$, las guías actuales no recomiendan la utilización de un modo de visión sobre otro; sin embargo, sí hacen énfasis en la velocidad a la que se debe de realizar la lectura, recomendando una velocidad de 10 a 15 CPS en modo sencillo y de 20 CPS en modos de visión múltiple ${ }^{3}$, esto debido a que una lectura a mayor velocidad se pudiera traducir en pasar por alto hallazgos con la consiguiente disminución de la tasa diagnóstica. Por último se cumplió también con una retención $<2 \%$ de las CE la cual es la complicación más común durante la realización de $\mathrm{CE}$, con frecuencias reportadas del 0.3 al $3 \%$, teniendo que tener en mente otras complicaciones clínicas (retención, aspiración del dispositivo a vía aérea, interferencia con dispositivos cardiacos) o técnicas (lagunas en la grabación, duración corta de la batería, falta de activación de la cápsula, falla de descarga, etc. $)^{9}$ reportadas con el uso de CE.

Los IC con los que no se logró cumplir fueron el tiempo de envío a CE en un episodio de HTDM, la documentación de la limpieza intestinal y el envío a EAD, fallando por lo tanto en 2 IC clasificados como mayores y uno clasificado como menor. El tiempo de envío a CE en un episodio de HTDM es importante porque se ha demostrado que un envío dentro de los primeros 14 días a CE se traduce en mejores tasas diagnósticas, comprobando hallazgos positivos en el $94 \%$ de las cápsulas realizadas tempranamente vs. el $34 \%$ en aquellas realizadas más allá de 14 días del último episodio de sangrado ${ }^{10}$; debido a esto se recomienda que el envío a CE se realice de forma temprana en $\geq 90 \%{ }^{3}$. En nuestro centro se realizó un envío oportuno a CE en el $63.76 \%$ de los casos, esto probablemente influenciado por que parte de las CE realizadas nos son referidas de hospitales regionales, con la consiguiente demora por trámites administrativos, así como por la no documentación en el reporte del tiempo transcurrido entre el último episodio de sangrado y la realización de la CE. En cuanto a la documentación de la limpieza intestinal, una adecuada visualización de la mucosa es fundamental para la detección de patologías intestinales, disminuye la necesidad de repetir la CE y de realizar otras maniobras por una inadecuada visualización durante el estudio; el uso o no de preparación intestinal, así como el tipo, sigue siendo controvertido, por lo cual se decidió marcar como punto de calidad el grado de limpieza intestinal obtenido mediante el uso de escalas validadas (Park, Brotz, etc.), independientemente de la maniobra utilizada, debiendo obtener una adecuada visualización en $\geq 95 \%$ de las $C^{3,11}$. En nuestro centro no se utilizaba previo a la publicación de la ESGE escalas validadas en el reporte endoscópico, por lo que se falló en el cumplimiento de dicho IC. En cuanto al envío adecuado a EAD, la realización de CE previa, la realización de EAD en pacientes con HTDM aumenta considerablemente la tasa diagnóstica y terapéutica de la EAD $(75 \%$ de encontrar hallazgos en la CE vs. $27.5 \%$ en aquellos con $\mathrm{CE}$ negativa $)^{12}$, esto útil también en otras indicaciones de $C E$, además, la CE puede orientar la ruta más adecuada de acceso, vía oral o rectal, por lo cual ambas técnicas se complementan. En nuestro centro se tuvo apego en el $63.01 \%$ de los casos de la meta mínima del $75 \%$, si bien es probable que se haya referido a un mayor número de pacientes de manera adecuada a la EAD, el problema constituyó una falta de documentación en el reporte de la CE de dicha recomendación. En cuanto al IC excluido, la identificación de pacientes con alto riesgo de retención de CE, es de suma importancia al ser la complicación más frecuente y al ser variable según el grupo en el que se realice; siendo desde el $2 \%$ cuando la CE se realiza por HTDM hasta el 130 $16 \%$ en EC o presencia de síntomas obstructivos sucesivamente, por lo tanto, debemos identificar al grupo de población de alto riesgo de retención de CE (EC conocida o sospechada, uso crónico de antiinflamatorios no esteroideos, resecciones intestinales, síntomas obstructivos, etc. $)^{13}$ y realizar a estos cápsula Patency; sin embargo, su disponibilidad no es amplia y no se cuenta con esta en el cuadro básico del sistema de salud mexicano, por lo que en nuestro centro no se realizó cápsula $\mathrm{Pa}$ tency, sustituyendo esta en los pacientes con alto riesgo con estudios de imagen (transito intestinal, 
enterografía por tomografía computarizada o enterografía por resonancia magnética) como válvula de seguridad, debido a que estas han reportando sensibilidad comparable a la cápsula Patency ${ }^{14}$.

Este es el primer estudio mexicano que evalúa la calidad de la CE en la valoración de IC. Es importante señalar que la interpretación de la mayoría de los estudios de CE incluidos en este trabajo se realizaron previamente a la existencia de la guía utilizada para la evaluación.

\section{Conclusiones}

Los IC son herramientas útiles para mejorar el desempeño de los estudios endoscópicos. En nuestra revisión se cumplieron 6 de los 9 indicadores de CE mencionados; cumpliendo con 4 de 6 de los IC clasificados como mayores y con 2 de 3 de los menores.

\section{Agradecimientos}

Gracias a todo el personal del Departamento de Endoscopia Gastrointestinal que hizo posible este estudio.

\section{Financiamiento}

No se ha recibido apoyo financiero para la realización del presente estudio, todo el material utilizado forma parte del Departamento de Endoscopia Gastrointestinal.

\section{Conflicto de intereses}

Los autores declaran no tener ningún conflicto de interés.

\section{Responsabilidades éticas}

Protección de personas y animales. Los autores declaran que para esta investigación no se han realizado experimentos en seres humanos ni en animales.

Confidencialidad de los datos. Los autores declaran que en este artículo no aparecen datos de pacientes.

Derecho a la privacidad y consentimiento informado. Los autores declaran que en este artículo no aparecen datos de pacientes.

\section{Bibliografía}

1. Rutter MD, Senore C, Bisshops R, Domagk D, Valori R, Kaminski MF, et al. The European Society of Gastrointestinal Endoscopy Quality Improvement Initiative: developing performance measures. United European Gastroenterol J. 2015;4:30-41.

2. Shim KN, Jeon SR, Jang HJ, Kim J, Lim YJ, Kim KO, et al. Quality indicators for small bowel capsule endoscopy. Clin Endosc. 2017;50:148-60.

3. Spada C, McNamara D, Despott EJ, Adler S, Cash BD, Fernández-Urién I, et al. Performance measures for small-bowel endoscopy. European Society of Gastrointestinal Endoscopy (ESGE) Quality Improvement Initiative. Endoscopy. 2019;51(6):574-98.

4. Delvaux M, Gay G. Capsule endoscopy: Technique and indications. Best Pract Res Clin Gastroenterol. 2008;22:813-37.

5. Wang A, Banerjee S, Barth BA, Bhat YM, Chauhan S, Gottlieb KT, et al. Wireless capsule endoscopy. Gastrointest Endosc. 2013;78:805-15.

6. Mehdizadeh S, Chen GC, Barkodar L, Enayati PJ, Pirouz S, Yadegari M, et al. Capsule endoscopy in paients with Crohn's disease: diagnostic yield and safety. Gastrointest Endosc. 2010;71:121-7.

7. Korman LY, Delvaux M, Gay G, Hagenmuller F, Keuchel M, Friedman S, et al. Capsule endoscopy structured terminology. Endoscopy. 2005;37:951-9.

8. Nakamura M, Murino A, O'Rourke A, Fraser C. A critical analysis of the effect of view mode and frame rate on reading time and lesion detection during capsule endoscopy. Dig Dis Sci. 2015;60:1743-7.

9. Ho KK, Joyce AM. Complications of capsule endoscopy. Gastrointest Endosc Clin N Am. 2007;17:169-78.

10. Bresci G, Parisi G, Bertoni M, Tumino E, Capria A. The role of video capsule endoscopy for evaluating obscure gastrointestinal bleeding: usefulness of early use. J Gastroenterol. 2005;40:256-9.

11. Park SC, Keum B, Hyun JJ, Seo YS, Kim YS, Jeen YT, et al. A novel cleansing score system for capsule endoscopy. World J Gastroenterol. 2010;16:875-80.

12. Teshima CW, Kuipers EJ, van Zanten SV, Mensink PBF. Double balloon enteroscopy and capsule endoscopy for obscure gastrointestinal bleeding: an updated meta-analysis. J Gastroenterol Hepatol. 2011;26:796-80.

13. Römmele C, Brueckner J, Messmann H, Gölder SK. Clinical experience with the PillCam Patency Capsule prior to Video Capsule Endoscopy: A real-world experience. Gastroenterol Res Pract. 2016;2016:9657053.

14. Mitselos IV, Katsanos K, Tsianos EV, Eliakim R, Christodoulou D. Clinical use of patency capsule: A comprehensive review of the literature. Inflamm Bowel Dis. 2018;24(11):2339-47. 